\title{
A Signal Processing View of Differintegration
}

\author{
Manuel D. Ortigueira, \\ Instituto Superior Técnico and UNINOVA ${ }^{1}$ \\ Campus da FCT da UNL, Quinta da Torre, \\ 2825 - 114 Monte da Caparica, Portugal \\ Tel. +351212948520 \\ E-mail: $\underline{\text { mdo@uninova.pt }}$
}

Keywords: Fractional derivative, differintegration, Bsplines..

\begin{abstract}
The fractional differintegration problem is treated from the Signal Processing point of view. A brief review of the Laplace transform approach to differintegration is done. The continuoustimeldiscrete-time system conversion is discussed and presented a Grünwald-Letnikov integration.
\end{abstract}

\section{Introduction}

The fractional calculus is nearly 300 years old. In fact, in a letter to Leibnitz, Bernoulli put him a question about the meaning of a non-integer derivative order. It was the beginning of a discussion about the theme that involved other mathematicians like: L' Hôpital, Euler and Fourier[5,6,12,13]. However, we can reference the beginning of the fractional calculus in the works of Liouville and Abel. Similarly, Liouville did several attempts. In one of them he presented a formula for fractional integration similar to $(1.1)$ :

$\mathrm{D}^{-\mathrm{p}} \varphi(\mathrm{t})=\frac{1}{(-1)^{\mathrm{p}} \Gamma(\mathrm{p})} \int_{0}^{\infty} \varphi(\mathrm{t}+\tau) \tau^{\mathrm{p}-1} \mathrm{~d} \tau-\infty<\mathrm{t}<\infty, \operatorname{Re}(\mathrm{p})>0$

where $\Gamma(\mathrm{p})$ is the gamma function. To this integral, with the term $(-1)^{\mathrm{p}}$ omitted, we give the name of Liouville's fractional integral. In other papers, Liouville went ahead with the development of ideas concerning this theme, having presented a generalization of the notion of incremental ratio to define a fractional derivative. This idea was recovered, later, by Grünwald (1867) and Letnikov (1868).

Riemann reached to an expression similar to (1.1) for the fractional integral

\footnotetext{
${ }^{1}$ Also with INESC, R. Alves Redol, 9, 2º 1000 - 029 Lisbon
}

and Arnaldo G. Batista

Departamento de Física da FCT/UNL

Campus da FCT da UNL, Quinta da Torre, 2825 - 114 Monte da Caparica, Portugal Tel. +351212948576

E-mail: $\underline{\text { agb@mail.fct.unl.pt }}$

$$
\mathrm{D}^{-\alpha} \varphi(\mathrm{t})=\frac{1}{\Gamma(\alpha)} \int_{0}^{\mathrm{t}} \frac{\varphi(\tau)}{(\mathrm{t}-\tau)^{1-\alpha}} \mathrm{d} \tau, \quad \mathrm{t}>0
$$

that, together with (1.2), became the more important basis for the fractional integration. It suits to refer that both Liouville and Riemann dealt with the called "complementary" functions that appear when we treat the differentiation of order $\alpha$ as an integration of order $-\alpha$. Holmgren (1865/66) and Letnikov (1868/74) discussed that problem when looking for the solution of differential equations, putting in a correct statement the fractional differentiation as inverse operation of the fractional integration. Besides, Holmgren gave a rigorous proof of Leibnitz' rule for the fractional derivative of the product of two functions. In the advent of $\mathrm{XX}^{\text {th }}$ century, Hadamard proposed a method of fractional differentiation with basis on the differentiation of Taylor's series associated with the function. Weyl (1917) defined a fractional integration suitable to periodic functions particular cases of Liouville and Riemann ones but that are, nowadays, the basis for fractional integration in R. An important contribution to the fractional differentiation was given by Marchaud (1927).

Nowadays, the unified formulation of differentiation and integration - called differintegration - based on Cauchy integral $[2,5,9,13]$ gained great popularity.

These developments allowed the study and solutions of fractional differential equations $[6,9,13]$ and a first approach into the formulation of the fractional linear system theory [10]. However, we remained without satisfactory answers for some questions, namely tied up with the conversion from continuous-time to discrete-time linear systems $[7,8,16]$. In this paper we will make a brief description of a Signal Processing route through Fractional Calculus and some questions are placed and answers proposed. In section 2 we revise the Laplace Transform based fractional differintegration and discuss the foundation of the Grünwald-Letnikov derivative suitable for numerical computations. In Section 3 we address the conversion from continuous-time to discrete-time problem from a very general perspective. In passing we suggest a definition of a 
Grünwald-Letnikov integration. The interest of splines in Approximation and Signal Processing $[14,15]$ lead us to treat this subject, by proposing new fractional splines. At last, we present some conclusions.

\section{The Laplace Transform approach}

\subsection{Obtaining the differintegration operator}

In the previous section, we made a brief introduction to the fractional calculus. We were not exhaustive in the sense that there are several other definitions of fractional differintegration we did not consider here. However, it seems to be clear to exist an inherent difficulty in obtaining a definition with enough generality and compatibility with the usual Signal Processing practice. In fact, in Signal Processing, we frequently assume that the signals have $\mathbf{R}$ as domain and use the Bilateral Laplace (LT) and Fourier (FT) Transforms. With these tools, the remarkably important Transfer Function and Frequency Response concepts are defined, with properties we want to preserve in the fractional case. These considerations led us to start from the transform point of view in order to generalise to the fractional case well-known properties of the Laplace Transform. For example, if $\alpha$ is a real number we expect to obtain:

$\operatorname{LT}\left[f^{(\alpha)}(t)\right]=s^{\alpha} F(s)$

where $F(s)$ is the LT of $f(t)$. This would be the generalisation of the usual property of the LT of the derivative or the integral. As we wish to work without unnecessary limitations and constraints we will be working in the context of the Generalised Functions, by considering exponential order and tempered distributions.

Instead of starting with the definition of differintegration, we invert the problem and take the LT as starting point since the expression (2.1) must be valid for any real $\alpha$. Essentially, the objective is to prolong the sequence:

$\ldots \mathrm{s}^{-\mathrm{n}} \ldots, \mathrm{s}^{-2}, \mathrm{~s}^{-1}, 1, \mathrm{~s}^{1}, \mathrm{~s}^{2}, \ldots, \mathrm{s}^{\mathrm{n}} \ldots$

in order to include other kind of exponents: rational or, generally, real and even complex numbers. We immediately observe that there are two ways for this extension to be obtained, depending on the LT region convergence: the left half-plane or the right half-plane. To solve our problem, we are going to perform the LT inversion of the function $\mathrm{s}^{-v}$. For now, we will consider the case $0<v<1$ and assume that its region of convergence is defined by $\operatorname{Re}(s)>0$ - causal case. This inversion can be obtained through the use of a real inversion formula for the Laplace Transform [4]. It gives:

$\delta_{+}^{(-v)}(\mathrm{t})=\frac{\mathrm{t}^{\mathrm{v}-1}}{\Gamma(\mathrm{v})} \mathrm{u}(\mathrm{t})$

which is exactly a generalization of the expression we obtain easily for negative integer values. The anti-causal case be treated similarly, but we only have to remember that LT[x(t)] $=X(-s)$ to conclude easily that:

$$
\delta_{-}^{(-v)}(t)=-\frac{t^{v-1} u(-t)}{\Gamma(v)}
$$

With (2.3) and (2.4), we can define $v$ order integrated of $x(t)$, $\mathrm{x}_{+}^{(-v)}(\mathrm{t})$ and $\mathrm{x}_{-}^{(-v)}(\mathrm{t})$, as the convolution of $\mathrm{x}(\mathrm{t})$ with $\delta_{+}^{(-v)}(\mathrm{t})$ and $\delta^{(-v)}(\mathrm{t})$ :

$x_{+}^{(-v)}(t)=\frac{1}{\Gamma(v)} \int_{-\infty}^{t} x(\tau) \cdot(t-\tau)^{v-1} d \tau$

and

$x_{-}^{(-v)}(t)=-\frac{1}{\Gamma(v)} \int_{t}^{\infty} x(\tau) \cdot(t-\tau)^{v-1} d \tau$

In the following we will consider the causal case, only. With the described procedure, we found out a generalisation of the primitive concept for orders $v \in] 0,1[$. To obtain similar results for every $\alpha \in \mathrm{R}$ we must proceed in order to generalise also the known properties of the primitive and derivative, namely that:

$D^{\alpha}\left\{D^{\beta}\right\}=D^{\alpha+\beta}=D^{\beta}\left\{D^{\alpha}\right\}$

should be valid for every $\alpha, \beta \in \mathrm{R}$. In particular, if $\mathrm{n}$ is a positive integer and $0<\mu<1$,

$D^{n}\left\{D^{\mu}\right\}=D^{\mu}\left\{D^{n}\right\}=D^{n+\mu}$

and

$\mathrm{D}^{-\mathrm{n}}\left\{\mathrm{D}^{-\mu}\right\}=\mathrm{D}^{-\mu}\left\{\mathrm{D}^{-\mathrm{n}}\right\}=\mathrm{D}^{-\mathrm{n}-\mu}$

Putting $\alpha=n+v, n \in N$ and $0<v<1,-\alpha=-n-v$ and using (2.5) we obtain:

$\mathrm{x}_{+}^{(-\alpha)}(\mathrm{t})=\frac{1}{\Gamma(v)} \mathrm{D}^{-\mathrm{n}}\left[\int_{-\infty}^{\mathrm{t}} \mathrm{x}(\tau) \cdot(\mathrm{t}-\tau)^{v-1} \mathrm{~d} \tau\right]$

But from the properties of the convolution $D^{n}[f * g]=\left[D^{n} f\right] * g$, for every $n \in Z$, we conclude easily that:

$\mathrm{x}_{+}^{(-\alpha)}(\mathrm{t})=\frac{1}{\Gamma(v)} \int_{-\infty}^{\mathrm{t}} \mathrm{x}^{(-\mathrm{n})}(\tau) \cdot(\mathrm{t}-\tau)^{\mathrm{v}-1} \mathrm{~d} \tau$

But as $v=\alpha-n$, we obtain:

$\mathrm{x}_{+}^{(-\alpha)}(\mathrm{t})=\frac{1}{\Gamma(\alpha-n)} \int_{-\infty}^{\mathrm{t}} \mathrm{x}^{(-\mathrm{n})}(\tau) \cdot(\mathrm{t}-\tau)^{\alpha-\mathrm{n}-1} \mathrm{~d} \tau$

where $\mathrm{n}$ is the integer part of $|\alpha|$. Equation (2.12) generalises (2.5) for every negative real order. To treat the derivative case, or $\alpha=n+v, n \in N$ and $0<v<1$, we have $\alpha=n+1-1+v=n+1$ (1-v), we have:

$x_{+}^{(\alpha)}(t)=\frac{1}{\Gamma(1-v)} D^{(n+1)}\left[\int_{-\infty}^{t} x(\tau) \cdot(t-\tau)^{-v} d \tau\right]$

and again:

$\mathrm{x}_{+}^{(\alpha)}(\mathrm{t})=\frac{1}{\Gamma(1-v)}\left[\int_{-\infty}^{\mathrm{t}} \mathrm{x}^{(\mathrm{n}+1)}(\tau) \cdot(\mathrm{t}-\tau)^{-v} \mathrm{~d} \tau\right]$

As $v=\alpha-n$, we obtain: 
$x_{+}^{(\alpha)}(t)=\frac{1}{\Gamma(-\alpha+n+1)}\left[\int_{-\infty}^{t} x^{(n+1)}(\tau) \cdot(t-\tau)^{-\alpha+n} d \tau\right]$

that is known as Caputo derivative [13]. Of course, $\operatorname{LT}\left[\mathrm{x}_{+}^{(\alpha)}\right.$

$(t)]=s^{\alpha} X(s)$, for $\operatorname{Re}(s)>0$. As referred in [12], this definition of fractional derivative has advantages over other approaches when we are dealing with fractional linear systems where we need non-zero initial conditions.

We may ask for reasons for not having computed the derivative in $\mathrm{t}^{-\mathrm{v}-1}$ instead of $\mathrm{x}(\mathrm{t})$. The answer is in the fact that $\mathrm{t}^{-\mathrm{v}-1}$ is one solution of the equation $\mathrm{t}^{\mathrm{v}+1} \cdot \mathrm{f}(\mathrm{t})=1$, that has infinite solutions with the general format $f(t)=t^{-v-1}+A . \delta(t)$, where $A \in R$. So, it cannot be considered a distribution in the sense used in [3]. Of course, we can consider the so-called finite part of that function, $\mathrm{t}^{-\mathrm{v}-1}$. If we do so and perform the differintegration operation in this function in (2.10) and (2.13) we obtain, after some manipulation:

$\mathrm{x}_{+}^{(\alpha)}(\mathrm{t})=\frac{1}{\Gamma(-\alpha)} \int_{-\infty}^{\mathrm{t}} \mathrm{x}(\tau) \cdot(\mathrm{t}-\tau)^{-\alpha-1} \mathrm{~d} \tau$

that generalises (2.5) for any $\alpha \in \mathrm{R}$. A similar procedure based on (2.6) would lead to:

$\mathrm{x}_{-}^{(\alpha)}(\mathrm{t})=-\frac{1}{\Gamma(v)} \int_{\mathrm{t}}^{\infty} \mathrm{x}(\tau) \cdot(\mathrm{t}-\tau)^{-\alpha-1} \mathrm{~d} \tau$

Equations (2.16) and (2.17) express respectively the socalled Riemann-Liouville and Weyl fractional derivatives $[6,13]$.

\subsection{The first difference and the Grünwald- Letnikov derivative}

Consider the discrete-time system having Transfer Function given by:

$\mathrm{H}(\mathrm{z})=\left(\frac{1-\mathrm{z}^{-1}}{\mathrm{~h}}\right)^{\alpha}$

The discrete-time equation corresponding to (2.1) can be described by a fractional order difference equation:

$\mathrm{y}_{\mathrm{n}}=(1-\mathrm{D})^{\alpha} \mathrm{x}_{\mathrm{n}}$

where is the unit delay operator. The corresponding Impulse Response is:

$\mathrm{h}_{\mathrm{n}}=(-1)^{\mathrm{n}}\left(\begin{array}{l}\alpha \\ \mathrm{n}\end{array}\right) \mathrm{u}_{\mathrm{n}}$

where $\left(\begin{array}{c}\alpha \\ n\end{array}\right)=\frac{\Gamma(\alpha+1)}{\Gamma(\alpha-n+1) \cdot n !}$ are the binomial coefficients. From the point of view of the stability of the system $\left(1-\mathrm{z}^{-1}\right)^{\alpha}$, we can say that if $\alpha$ is a negative integer, it is unstable, in general, excepting the case $\alpha=-1$ (accumulator); in this situation, the system is wide sense stable. If $\alpha$ is a positive integer, it is a FIR, so it is always stable in every sense. If $\alpha>0$, the system will be stable in any sense; if $-1<\alpha<0$, the system will be wide sense stable, since the impulse response goes slowly to zero, $\mathrm{h}_{\mathrm{n}} \approx \mathrm{c} . \mathrm{n}^{-\alpha-1}$. We conclude that if $|\alpha|<1$, the system is, at least, wide sense stable. Now, we define a fractional (discrete-time) stochastic process as the output of a fractional system excited by stationary white noise:

$\mathrm{x}(\mathrm{k})=\mathrm{h}(\mathrm{k}) * \mathrm{n}(\mathrm{k})$

where $n(k)$ is a stationary white noise with variance $\sigma^{2}$. We define, as usually, the autocorrelation function by:

$\mathrm{R}_{\mathrm{x}}(\mathrm{k})=\sigma^{2} \sum_{\mathrm{i}=0}^{\infty} \mathrm{h}_{\mathrm{i}}^{*} \cdot \mathrm{h}_{\mathrm{i}+\mathrm{k}} \quad \mathrm{k} \geq 0$

The computation of its autocorrelation function is slightly involved [11] and is given by:

$\mathrm{R}_{\mathrm{x}}(\mathrm{k})=\sigma^{2}(-1)^{\mathrm{k}} \frac{\Gamma(1+2 \alpha)}{\Gamma(\alpha+\mathrm{k}+1) \Gamma(\alpha-\mathrm{k}+1)}$

if $1+2 \alpha>0$, or $\alpha>-1 / 2$. If $\alpha$ non integer and $\alpha<-1 / 2$, we obtain a negative power, meaning that, in such situation, the finite difference cannot be used. The Grünwald-Letnikov approach to fractional derivative consists in making the sampling interval $h$ go to zero in (2.18). Letting $z=e^{\text {sh }}$ we have:

$\frac{\left(1-\mathrm{e}^{-\mathrm{sh}}\right)^{\alpha}}{\mathrm{h}^{\alpha}}=\frac{1}{\mathrm{~h}^{\alpha}} \sum_{\mathrm{k}=0}^{\infty}(-1)^{\mathrm{k}}\left(\begin{array}{l}\alpha \\ \mathrm{k}\end{array}\right) \mathrm{e}^{-\mathrm{shk}}$

if $\mathrm{h}>0$ and $\operatorname{Re}(\mathrm{s})>0$. Similarly

$\frac{\left(\mathrm{e}^{\mathrm{sh}}-1\right)^{\alpha}}{\mathrm{h}^{\alpha}}=\frac{(-1)^{\alpha}}{\mathrm{h}^{\alpha}} \sum_{\mathrm{k}=0}^{\infty}(-1)^{\mathrm{k}}\left(\begin{array}{l}\alpha \\ \mathrm{k}\end{array}\right) \mathrm{e}^{\text {shk }}$

provided that $\mathrm{h}>0$ and $\operatorname{Re}(\mathrm{s})<0$. The first members in (2.24) and (2.25) converge to $\mathrm{s}^{\alpha}$ with $\mathrm{h} \rightarrow 0^{+}$. These expressions, when inverted back into time lead, respectively, to

$\mathrm{d}_{+}^{(\alpha)}(\mathrm{t})=\lim _{\mathrm{h} \rightarrow 0^{+}} \frac{1}{\mathrm{~h}^{\alpha}} \sum_{\mathrm{k}=0}^{\infty}(-1)^{\mathrm{k}}\left(\begin{array}{l}\alpha \\ \mathrm{k}\end{array}\right) \delta(\mathrm{t}-\mathrm{kh})$

if $\operatorname{Re}(s)>0$, and

$\mathrm{d}_{-}^{(\alpha)}(\mathrm{t})=\lim _{\mathrm{h} \rightarrow 0^{+}} \frac{(-1)^{\alpha}}{\mathrm{h}^{\alpha}} \sum_{\mathrm{k}=0}^{\infty}(-1)^{\mathrm{k}}\left(\begin{array}{l}\alpha \\ \mathrm{k}\end{array}\right) \delta(\mathrm{t}+\mathrm{kh})$

provided that $\operatorname{Re}(s)<0\left({ }^{d}\right)$. The convolution of (2.26) and (2.27) with a given signal, $f(t)$, leads to the GrünwaldLetnikov backward and forward derivatives.

\section{Definition 2.1}

Let $f(t)$ a limited function and $\alpha>0$. We define derivative of order $\alpha$, backward and forward, respectively, by

$$
f_{+}^{(\alpha)}(t)=\lim _{h \rightarrow 0^{+}} \frac{\sum_{k=0}^{\infty}(-1)^{k}\left(\begin{array}{l}
\alpha \\
k
\end{array}\right) f(t-k h)}{h^{\alpha}}
$$

or

\footnotetext{
1 We used d instead $\delta$, to avoid confusion, while the equivalence is not proved.
} 
$\mathrm{f}_{-}^{(\alpha)}(\mathrm{t})=\lim _{\mathrm{h} \rightarrow 0^{+}}(-1) \frac{\sum_{\mathrm{k}=0}^{\infty}(-1)^{\mathrm{k}}\left(\begin{array}{l}\alpha \\ \mathrm{k}\end{array}\right) \mathrm{f}(\mathrm{t}+\mathrm{kh})}{\mathrm{h}^{\alpha}}$

We can show [14] that $\left|\left(\begin{array}{l}\alpha \\ \mathrm{k}\end{array}\right)\right| \leq \frac{\mathrm{c}}{\mathrm{k}^{1+\alpha}}$ with $\alpha$ kept constant and $\mathrm{k} \rightarrow \infty$. This condition shows that the series $\mathrm{C}(\alpha)=$ $\sum_{0}^{\infty}\left|\left(\begin{array}{l}\alpha \\ k\end{array}\right)\right|$ is absolutely convergent and assuring, thus, that the series (2.28) and (2.29) converge absolutely and uniformly for limited functions provided that $\alpha>0$ [13]. In this case, it is not hard to prove the equivalence of (2.16) and (2.28) - or (2.17) and (2.29) [12]. On the other hand (2.23) implies that the previous series converge also for $\alpha>-1 / 2$ for square integrable functions. For other cases, the definitions (2.28) and (2.29) may not to remain valid, since, in general, they are divergent. Such happens, for example, if $f(t)=1$ or $\mathrm{f}(\mathrm{t})$ is periodic. It is noteworthy that it can exist a left derivative without existing the right one and vice-versa. To see why, let us apply both definitions to the function $\mathrm{f}(\mathrm{t})=$ $\mathrm{e}^{\mathrm{st}}$. If $\operatorname{Re}(\mathrm{s})>0$, the $\mathrm{f}^{\mathrm{t}}$ converges to $\mathrm{s}^{\alpha} \cdot \mathrm{e}^{\mathrm{st}}$, while the $2^{\mathrm{d}}$ diverges; if $\operatorname{Re}(s)<0$, we have the reverse situation. On the other hand, in the limit computation, a change from $h$ to $-h$ interchanges (2.28) and (2.29). It is somehow difficult to accept that a local property of a function has such behaviour at all the points. To avoid this a generalisation of such expressions to use left and right points is proposed in [14].

\section{The s2z conversion}

\subsection{The continuous-time to discrete-time conversion problem}

The most common and useful linear systems are described by linear fractional differential equations. Here, it will be assumed that the coefficients of the equation are constant, so the corresponding system will be a fractional linear time-invariant (FLTI) system described by a differential equation with the format:

$\sum_{i=0}^{N} a_{i} D^{i v} y(t)=\sum_{j=0}^{M} b_{j} D^{j v} x(t)$

where $\mathrm{D}$ is the derivation operator and the $\mathrm{v}$ is a positive real $\left({ }^{1}\right)$. If we apply the LT to the equation (3.1) we obtain:

$$
\mathrm{H}(\mathrm{s})=\frac{\sum_{\mathrm{j}=0}^{\mathrm{M}} \mathrm{b}_{\mathrm{j}} \mathrm{s}^{\mathrm{j} v}}{\sum_{\mathrm{i}=0}^{\mathrm{N}} \mathrm{a}_{\mathrm{i}} \mathrm{s} v}
$$

1 We do not need to be so restrictive with the differintegration orders $\{$ see $[13,14]\}$. which is the Transfer Function. We will assume that $\mathrm{M}<\mathrm{N}$.

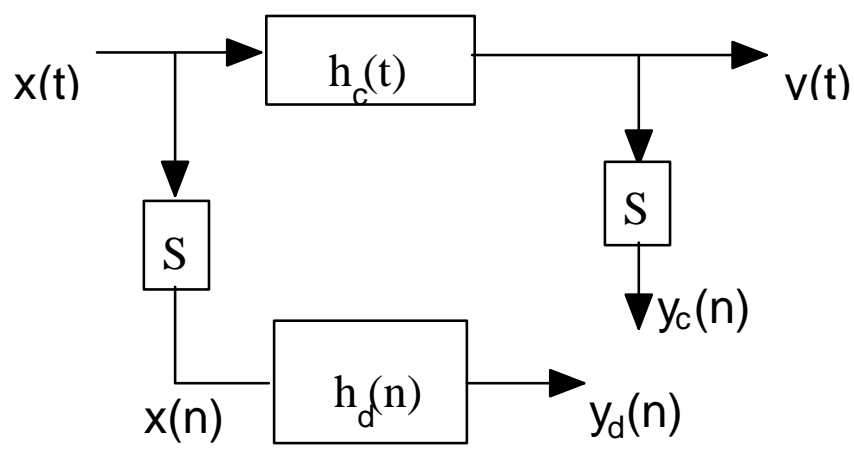

\section{S - Sampler}

fig. 1 equivalence between continuous and discrete linear systems

Usually, in Signal Processing applications, most times, we are interested in obtaining a discrete-time (DT) equivalent of the continuous-time (CT) system to:

a) simulate CT systems by DT means

b) process CT signals by DT systems

c) design DT systems by using the well-known CT design methods

d) modelling CT practical systems by using DT signals resulting from experimental measurements

In figure 1, we illustrate the process of constructing a discrete-time equivalent to a continuous-time system. This discrete-time equivalent will be described by a difference (eventually, fractional) equation. This means that we want to make a s to $\mathrm{z}(\mathrm{s} 2 \mathrm{z})$ conversion. The $\alpha=1$ case is well studied and several techniques to convert a differential equation into a difference equation $(\mathrm{s} 2 \mathrm{z})$ and vice-versa (z2s) were developed. Following [1] we can group the most important methods into:

a) Matching time responses (response-invariant transformation).

b) Matching terms in factored transfer function

c) Matching terms in partial fraction decomposition of the transfer function

d) Magnitude-invariant method

e) Conversion of the differential equation to a difference equation using difference operators.

f) Numerical solution of the differential equation by using numerical integration algorithms

g) Rational approximations to the exponential function.

The problem is that these procedures are not suitable for the $\alpha<1$ case.

\subsection{The first difference approach}

The first difference approach consists in making the substitution 
$\mathrm{s}^{\alpha}=\left(\frac{1-\mathrm{z}^{-1}}{\mathrm{~T}}\right)^{\alpha}=\frac{1}{\mathrm{~T}^{\alpha}} \sum_{\mathrm{n}=0}^{\infty}\left(\begin{array}{l}\alpha \\ \mathrm{n}\end{array}\right)(-1)^{\mathrm{n}} \mathrm{z}^{-\mathrm{n}}$

Inserting (3.3) into (3.2) we obtain the Transfer Function of the converted discrete-time system, that we can write as:

$\mathrm{H}(\mathrm{z})=\frac{\sum_{\mathrm{n}=0}^{\infty}\left[\sum_{j=0}^{M} \frac{1}{T^{j v}} b_{j}\left(\begin{array}{c}j v \\ n\end{array}\right)\right](-1)^{\mathrm{n}} \mathrm{z}^{-\mathrm{n}}}{\sum_{\mathrm{n}=0}^{\infty}\left[\sum_{j=0}^{N} \frac{1}{T^{j v}} a_{j}\left(\begin{array}{c}j v \\ n\end{array}\right)\right](-1)^{\mathrm{n}} \mathrm{z}^{-\mathrm{n}}}$

This leads us to conclude that the discrete-time equivalent is an ARMA system with infinite orders. In practical applications we have to truncate the outer summations, but we must have in mind that the truncation order must be very high, since the coefficients converge very slowly, equation (2.27). Besides this drawback, the approximation in (3.3) does not preserve the phase. In fact,

$\left(\frac{1-\mathrm{e}^{-\mathrm{j} \omega}}{\mathrm{T}}\right)^{\alpha}=\frac{1}{\mathrm{~T}^{\alpha}} \mathrm{e}^{-\mathrm{j} \omega \alpha / 2} \cdot(2 \mathrm{j})^{\alpha} \cdot \sin ^{\alpha}(\omega / 2)$

introducing a phase delay equal to $\omega \alpha / 2$. This leads to poor results even in the $\alpha=1$ case.

\subsection{A general theoretical approach}

From figure 1, it is clear that we may obtain different solutions for different input functions. The usual approach is based on the impulse or step responses. Although these functions are very important in applications, neither the impulse nor the step is satisfactory test functions, because the corresponding responses are very different from the inputs. This does not happen with the exponential function that is the eigenfunction of every linear system. Thus, letting $\mathrm{x}(\mathrm{t})=\mathrm{e}^{\mathrm{st}}$ and $\mathrm{x}_{\mathrm{t}}=\mathrm{z}^{\mathrm{n}}$ be the inputs to CT and DT systems, the outputs will be $y(t)=H_{c}(s) e^{\text {st }}$ and $y_{n}=H_{d}(z) \cdot z^{n}$. Let $\mathrm{z}=\mathrm{e}^{\mathrm{sT}}$. We say that a continuous-time system with transfer function $\mathrm{H}_{c}(\mathrm{~s})$ is equivalent to a discrete-time system with transfer function $\mathrm{H}_{\mathrm{d}}\left(\mathrm{e}^{\mathrm{sT}}\right)$, if

$\mathrm{H}_{\mathrm{d}}\left(\mathrm{e}^{\mathrm{sT}}\right)=\mathrm{H}_{\mathrm{c}}(\mathrm{s})$

However, $\mathrm{H}_{\mathrm{d}}\left(\mathrm{e}^{\mathrm{sT}}\right)$ is a periodic function with period equal to j. $2 \pi / T$. So, the equivalence will be restricted to the horizontal strip ST $=\left\{\mathrm{z}: \mathrm{z} \in \mathrm{C},|\operatorname{Im}(\mathrm{z})|<\frac{2 \pi}{\mathrm{T}}\right\}$. Let $\mathrm{H}_{\mathrm{d}}\left(\mathrm{e}^{\mathrm{sT}}\right)$ be equal to $\mathrm{H}_{\mathrm{c}}(\mathrm{s})$ inside the above strip. Then, we conclude easily that

$\mathrm{H}_{\mathrm{d}}\left(\mathrm{e}^{\mathrm{sT}}\right)=\sum_{-\infty}^{+\infty} \mathrm{H}_{\mathrm{c}}\left(\mathrm{s}+\mathrm{j} \frac{2 \pi}{\mathrm{T}} \mathrm{n}\right)$

To assure that (3.6) is valid, $\mathrm{H}_{\mathrm{c}}(\mathrm{s})$, must be a "strip-limited" function in the sense that it must be equal to zero outside the strip ST. As it is known, there are no realizable linear systems having a Transfer Function equal to zero, outside any strip. So, if we use $H_{c}$ in eq. (3.6), this will be verified only as an approximation that will become as better as $\mathrm{T}$ becomes smaller, if $\mathrm{H}_{\mathrm{c}}(\mathrm{s})$ goes to zero with increasing values if $\operatorname{Im}(\mathrm{s})$. As (3.7) represents a periodic function, it has a Fourier series:
$\mathrm{H}_{\mathrm{d}}\left(\mathrm{e}^{\mathrm{sT}}\right)=\sum_{-\infty}^{+\infty} \mathrm{h}_{\mathrm{dn}} \mathrm{e}^{-\mathrm{snT}}$

with Fourier coefficients given by:

$\mathrm{h}_{\mathrm{dn}}=\frac{\mathrm{T}}{2 \pi} \int_{-\pi \mathrm{j} / \mathrm{T}}^{\pi \mathrm{j} / \mathrm{T}} \mathrm{H}_{\mathrm{c}}(\mathrm{s}) \mathrm{e}^{\mathrm{snT}} \mathrm{ds}$

If $\mathrm{H}_{\mathrm{c}}(\mathrm{s})$ is nonzero only in the strip $|\operatorname{Im}(\mathrm{s})|<\pi / \mathrm{T}$, it is simple to conclude that

$\mathrm{h}_{\mathrm{dn}}=\mathrm{T} \cdot \mathrm{h}_{\mathrm{c}}(\mathrm{nT})$

Then, the impulse invariant conversion technique is an approximation to the eigenequivalence method. On the other hand, a $z=e^{s \mathrm{~T}}$ variable change in (3.9), we obtain:

$\mathrm{h}_{\mathrm{dn}}=\frac{1}{2 \pi \mathrm{j}} \int_{\mathrm{c}} \mathrm{H}_{\mathrm{d}}(\mathrm{z}) \mathrm{z}^{\mathrm{n}-1} \mathrm{dz}$

that is the usual inversion formula for the $\mathrm{Z}$ Transform.

In the $\mathrm{M}<\mathrm{N}$ case, the transfer function (3.2) can be considered as a sum of partial fractions of the form

$\mathrm{H}_{\mathrm{c}}(\mathrm{s})=\frac{1}{\left(\mathrm{~s}^{\mathrm{v}}-\mathrm{p}\right)^{\mathrm{k}}} \quad \mathrm{k}=1,2, \ldots$

We can then apply (3.7) to each fraction. Let $v=k=1$. In this case [4],

$H_{d}\left(e^{s T}\right)=\sum_{-\infty}^{+\infty} \frac{1}{s-p+j \frac{2 \pi}{T} n}=\frac{T}{2} \frac{1+e^{-(s-p) T}}{1-e^{-(s-p) T}}$

and

$\mathrm{H}_{\mathrm{d}}(\mathrm{z})=\frac{\mathrm{T}}{2} \frac{1+\mathrm{e}^{\mathrm{pT}} \mathrm{z}^{-1}}{1-\mathrm{e}^{\mathrm{pT}} \mathrm{z}^{-1}}$

when $\mathrm{p}=0$, we obtain the bilinear transformation that appears as the DT analogue of the integrator, allowing us to use the transformation (Tustin):

$\frac{1}{\mathrm{~s}}=\frac{\mathrm{T}}{2} \frac{1+\mathrm{z}^{-1}}{1-\mathrm{z}^{-1}}$

By integer differentiation of both sides in

$\sum_{-\infty}^{+\infty} \frac{1}{s-p+j \frac{2 \pi}{T} n}=\frac{T}{2} \frac{1+e^{-(s-p) T}}{1-e^{-(s-p) T}}$,

we obtain the conversions for the $v=1, k=2,3, \ldots$, cases. For example, for $\mathrm{k}=2$, we obtain:

$\frac{\mathrm{T}^{2} \mathrm{e}^{\mathrm{pT}} \cdot \mathrm{e}^{-\mathrm{sT}}}{\left(1-\mathrm{e}^{\mathrm{pT}} \mathrm{e}^{-\mathrm{sT}}\right)^{2}}=\sum_{-\infty}^{+\infty} \frac{1}{\left(s-p+j \frac{2 \pi}{\mathrm{T}} \mathrm{n}\right)^{2}}$

giving

$\mathrm{H}_{\mathrm{d}}(\mathrm{z})=\frac{\mathrm{T}^{2} \mathrm{e}^{\mathrm{pT}} \cdot \mathrm{z}^{-1}}{\left(1-\mathrm{e}^{\mathrm{pT}} \mathrm{z}^{-1}\right)^{2}}$

This procedure cannot be used for $\mathrm{H}(\mathrm{s})=\mathrm{s}$, for example, since this function is not strip-limited and the corresponding system is not stable. If $\mathrm{T}$ is small, the application of this kind of procedure did not show better performances than those we obtain by using the bilinear transformation (3.15). So, this has been widely used. However, we must firmly remark that (3.15) states an approximation to the integral and this is not equivalent that 
$\mathrm{s}=\frac{2}{\mathrm{~T}} \frac{1-\mathrm{z}^{-1}}{1+\mathrm{z}^{-1}}$ is an approximation to the derivative. Similarly in the non-fractional case, we would like to obtain the DT equivalent to $\frac{1}{\mathrm{~s}^{\alpha}}$, by computing

$\mathrm{D}^{\alpha-1}\left[\sum_{-\infty}^{+\infty} \frac{1}{\mathrm{~s}+\mathrm{j} \frac{2 \pi}{\mathrm{T}} \mathrm{n}}\right]=(-1)^{\alpha-1} \Gamma(\alpha) \cdot \sum_{-\infty}^{+\infty} \frac{1}{\left(\mathrm{~s}+\mathrm{j} \frac{2 \pi}{\mathrm{T}} \mathrm{n}\right)^{\alpha}}$, but it seems that $\mathrm{D}^{\alpha-1}\left[\frac{\mathrm{T}}{2} \frac{1+\mathrm{e}^{-\mathrm{sT}}}{1-\mathrm{e}^{-\mathrm{sT}}}\right]$ does not have a closed form.

\subsection{The Grünwald-Letnikov integral}

According to what we just said, when converting a continuous-time system to discrete-time, it is more common to use the bilinear transformation:

$\mathrm{s}=\frac{2}{\mathrm{~T}} \cdot \frac{1-\mathrm{z}^{-1}}{1+\mathrm{z}^{-1}}$

We must remark again that this equation must be more suitable for integral than derivative computation, since $\frac{2}{\mathrm{~h}} \frac{1+\mathrm{e}^{-\mathrm{sh}}}{1-\mathrm{e}^{-\mathrm{sh}}}$ is a better approximation to $\frac{1}{\mathrm{~s}}$ than $\frac{\mathrm{h}}{1-\mathrm{e}^{-\mathrm{sh}}}$; else, it has an important advantage over (3.3): the phase is $-90^{\circ}$. However, while being very useful in discrete-time modelling of the integrator it is not interesting for modelling the differentiator. Similarly, in fractional integration we can use

$\mathrm{s}^{\alpha}=\left(\frac{2}{\mathrm{~T}} \cdot \frac{1-\mathrm{z}^{-1}}{1+\mathrm{z}^{-1}}\right)^{\alpha}=\frac{2^{\alpha}}{\mathrm{T}^{\alpha}} \sum_{\mathrm{n}=0}^{\infty} \mathrm{C}_{\mathrm{n}}^{\alpha} \mathrm{z}^{-\mathrm{n}}$

where

$\mathrm{C}_{\mathrm{n}}^{\alpha}=\sum_{\mathrm{k}=0}^{\mathrm{n}}(-1)^{\mathrm{k}} \cdot\left(\begin{array}{l}\alpha \\ \mathrm{k}\end{array}\right)\left(\begin{array}{l}-\alpha \\ \mathrm{n}-\mathrm{k}\end{array}\right)$

are the convolution of he coefficients of two binomial series. With some manipulation we obtain

$\mathrm{C}_{\mathrm{n}}^{\alpha}=\alpha \cdot(-1)^{\mathrm{n}} \frac{1}{\mathrm{n} !} \sum_{\mathrm{k}=0}^{\mathrm{n}} \cdot\left(\begin{array}{l}\mathrm{n} \\ \mathrm{k}\end{array}\right)(\alpha-\mathrm{k}+1)_{\mathrm{n}-1}$
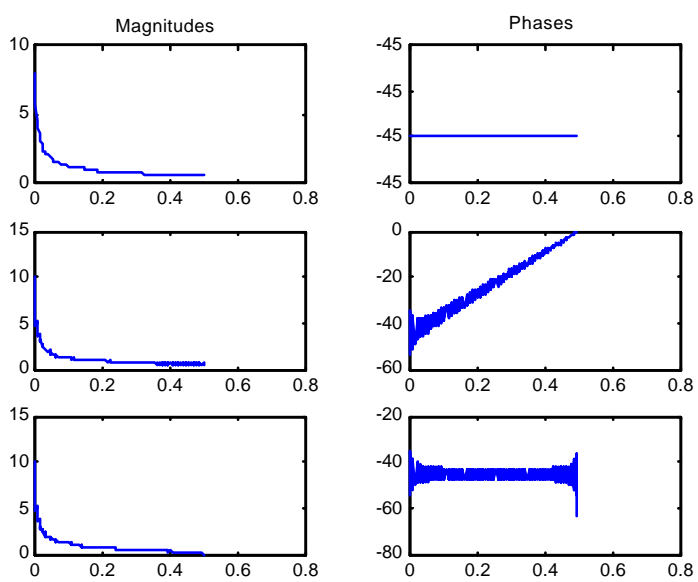

Figure 2 - Magnitudes and phases of the Frequency Responses corresponding to $\mathrm{\alpha}=-1 / 2$
The above considerations are illustrated in figures 2 and 3 obtained with $\alpha= \pm 1 / 2$. We computed 200 points of the impulse responses and computed their Fourier Transforms, represented in strips 2 and 3 in the pictures. In the first strip we present the continuous-time Fourier Transform $(j \omega)^{\alpha}$. According to the results, (3.19) must be used only in integration. On the other hand, we verified that the coefficients in (3.19) converge as similarly to the binomial coefficients when $\alpha$ is negative.
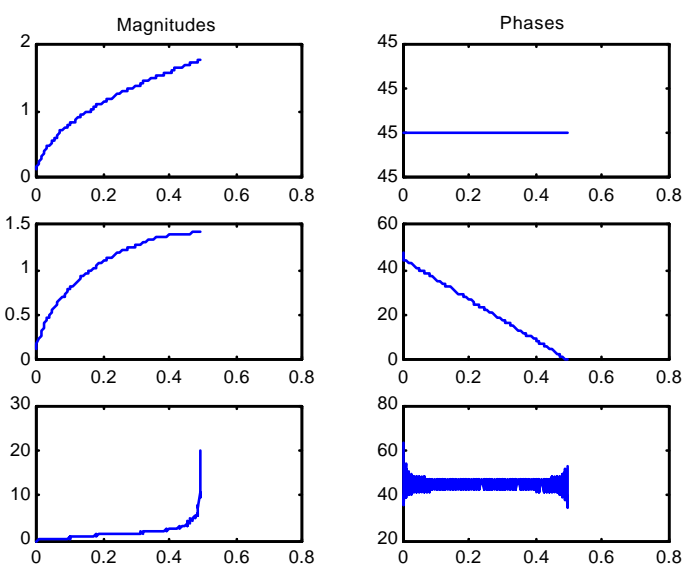

Figure 3 - Magnitudes and phases of the Frequency Responses corresponding to $\mathrm{O}=1 / 2$

So, we must transform (3.2) in order to make negative powers appear, by dividing numerator and denominator by $\mathrm{s}{ }^{\mathrm{K} v}$, with $\mathrm{K}=\max (\mathrm{M}, \mathrm{N})$. We will obtain:

$\mathrm{H}(\mathrm{z})=\frac{\sum_{\mathrm{n}=0}^{\infty}\left[\sum_{j=0}^{\mathrm{M}} \frac{2^{(\mathrm{j}-\mathrm{K}) v}}{\mathrm{~T}^{(\mathrm{j}-\mathrm{K}) v}} \mathrm{~b}_{\mathrm{j}} \mathrm{C}_{\mathrm{n}}^{(\mathrm{j}-\mathrm{K}) v}\right] \mathrm{z}^{-\mathrm{n}}}{\sum_{\mathrm{n}=0}^{\infty}\left[\sum_{\mathrm{j}=0}^{\mathrm{N}} \frac{2^{(\mathrm{j}-\mathrm{K}) v}}{\mathrm{~T}^{(\mathrm{j}-\mathrm{K}) v}} \mathrm{a}_{\mathrm{j}} \mathrm{C}_{\mathrm{n}}^{(\mathrm{j}-\mathrm{K}) v}\right] \mathrm{z}^{-\mathrm{n}}}$

that is the transfer function of the equivalent discrete-time system. Some of the research in this area has been directed towards obtaining rational approximations to (3.19) [16].

With (3.19) we can obtain similar results to the ones obtained with (3.3). In particular we can obtain a GrünwaldLetnikov like fractional integral:

$\mathrm{x}^{(\alpha)}(\mathrm{t})=\lim _{\mathrm{h} \rightarrow 0^{+}} \frac{2^{\alpha}}{\mathrm{h}^{\alpha}} \sum_{\mathrm{n}=0}^{\infty} \mathrm{C}_{\mathrm{n}}^{\alpha} \mathrm{x}(\mathrm{t}-\mathrm{nh}) \quad \alpha<0$

\section{On the fractional B-splines}

The signal processing with splines have been acquiring increasing interest due to its flexibility in interpolation, sampling and wavelet transform [15]. Recently, causal and symmetric fractional B-splines were proposed [16]. A closed look into the proposed B-spline definitions reveals that they seem strange. Here, we will face the problem and propose a new definition for the symmetric B-splines. 
A nth degree B-spline, $\beta_{0}^{\mathrm{n}}(\mathrm{t})$, is a symmetric function resulting from $\mathrm{n}$-fold convolution of the rectangle function $\beta_{0}^{0}(t)=\left\{\begin{array}{cc}1 & |t|<1 / 2 \\ 1 / 2 & |t|=1 / 2 \\ 0 & |t|>1 / 2\end{array}\right.$

Its LT is an analytic function given by

$\mathrm{B}_{0}^{\mathrm{n}}(\mathrm{s})=\left[\frac{\mathrm{e}^{\mathrm{s} / 2}-\mathrm{e}^{-\mathrm{s} / 2}}{\mathrm{~s}}\right]^{\mathrm{n}+1}=\frac{\mathrm{e}^{\mathrm{s}(\mathrm{n}+1) / 2}}{\mathrm{~s}^{\mathrm{n}+1}} \sum_{\mathrm{k}=0}^{\mathrm{n}+1}\left(\begin{array}{c}\mathrm{n}+1 \\ \mathrm{k}\end{array}\right)(-1)^{\mathrm{k}} \mathrm{e}^{-\mathrm{sk}}$

So, the corresponding FT exists and is given by:

$B_{0}^{n}(\omega)=\left[\frac{\sin (\omega / 2)}{\omega / 2}\right]^{n+1}=\frac{e^{j \omega(n+1) / 2}}{(j \omega)^{n+1}} \sum_{k=0}^{n+1}\left(\begin{array}{c}n+1 \\ k\end{array}\right)(-1)^{k} e^{-j \omega k}$

From (4.2), we obtain:

$\beta_{0}^{\mathrm{n}}(\mathrm{t})=\frac{1}{\mathrm{n} !} \sum_{\mathrm{k}=0}^{\mathrm{n}+1}\left(\begin{array}{c}\mathrm{n}+1 \\ \mathrm{k}\end{array}\right)(-1)^{\mathrm{k}}\left(\mathrm{t}-\mathrm{k}+\frac{\mathrm{n}+1}{2}\right)_{+}^{\mathrm{n}}$

On the other hand, as

$\mathrm{FT}^{-1}\left[(j \omega)^{-\mathrm{n}-1}\right]=\frac{1}{2} \frac{\mathrm{t}^{\mathrm{n}} \operatorname{sgn}(\mathrm{t})}{\mathrm{n} !}=\frac{\mathrm{t}_{0}}{\mathrm{n} !}$

we obtain, from (4.3) and (4.5):

$\beta_{0}^{\mathrm{n}}(\mathrm{t})=\frac{1}{\mathrm{n} !} \sum_{\mathrm{k}=0}^{\mathrm{n}+1}\left(\begin{array}{c}\mathrm{n}+1 \\ \mathrm{k}\end{array}\right)(-1)^{\mathrm{k}}\left(\mathrm{t}-\mathrm{k}+\frac{\mathrm{n}+1}{2}\right)_{0}^{\mathrm{n}}$

that seem to be different from (4.4), but due to the symmetry of the coefficients represents the same function.

Now, we are going to face the problem of defining a fractional symmetric B-spline. Let $0<v<1$ and consider the function:

$\mathrm{B}_{0}^{v}(\omega)=\left[\frac{\sin (\omega / 2)}{\omega / 2}\right]^{v}$

As $0<v<1,(-1)^{v / 2}$ is a complex number and then the inverse transform of will be a complex function. To avoid this, we consider the function:

$B^{v}(\omega)=\left|\frac{\sin (\omega / 2)}{\omega / 2}\right|^{v}$

As,

$\left|\frac{\sin (\omega / 2)}{\omega / 2}\right|^{v}=\lim _{s \rightarrow j \omega} \frac{\left[1-e^{-s}\right]^{\frac{v}{2}}\left[1-e^{s}\right]^{\frac{v}{2}}}{s^{v / 2} .(-s)^{v / 2}}$

It is clear, now, that the inverse transform of the previous function is given by the autocorrelation of the function:

$$
\beta_{+}^{v / 2}(\mathrm{t})=\frac{1}{\Gamma(\mathrm{v} / 2)} \sum_{\mathrm{k}=0}^{+\infty}\left(\begin{array}{c}
\mathrm{v} / 2 \\
\mathrm{k}
\end{array}\right)(-1)^{\mathrm{k}}(\mathrm{t}-\mathrm{k})_{+}^{\mathrm{v} / 2-1}
$$

To compute such autocorrelation, we have to compute the autocorrelation of the function $\delta^{(-v / 2)}(t)=\frac{(t)_{+}^{v / 2-1}}{\Gamma(v / 2)}$. This is easily computed giving:

$\left.R_{\delta}(t)=\frac{|t|^{v-1} \cdot B(v / 2,1-v)}{\Gamma^{2}(v)} \quad v \in\right] 0,1[$ where $\mathrm{B}(\mathrm{p}, \mathrm{q})$ is the Euler beta function. As $\mathrm{B}(\mathrm{p}, \mathrm{q})=$ $\frac{\Gamma(p) \cdot \Gamma(q)}{\Gamma(p+q)}$ and $\Gamma(v / 2) \cdot \Gamma(1-v / 2)=\frac{\pi}{\sin (v \pi / 2)}$ we obtain:

$\left.\mathrm{R}_{\delta}(\mathrm{t})=\frac{\Gamma(1-\mathrm{v}) \sin (\mathrm{v} \pi / 2)}{\pi}|\mathrm{t}|^{\mathrm{v}-1} \quad \mathrm{v} \in\right] 0,1[$

On the other hand [11], for every $\theta \in R$, but non-even integer

$\sum_{\mathrm{k}=0}^{\infty}\left(\begin{array}{c}\theta \\ \mathrm{k}\end{array}\right)\left(\begin{array}{c}\theta \\ \mathrm{k}+\mathrm{n}\end{array}\right)=\frac{\Gamma(1+\theta)}{\Gamma(\theta / 2+n+1) \Gamma(\theta / 2-n+1)}$

So, the continuous-time function corresponding to (4.8) is given by:

$b^{v}(t)=\sum_{k=-\infty}^{+\infty} \frac{\Gamma(1-v) \cdot \Gamma(1+v) \cdot \sin (v \pi / 2)(-1)^{k}}{\pi \Gamma(v / 2+k+1) \Gamma(v / 2-k+1)} \cdot|t-k|^{v-1}$

In [15], a symmetric B-spline is defined as inverse FT of the function:

$\mathrm{B}_{0}^{\alpha}(\omega)=\left|\frac{\sin (\omega / 2)}{\omega / 2}\right|^{\alpha+1}$

However, this definition has the disadvantage of giving a strange spline, when $\alpha$ is an even positive integer. To avoid this, we are going to present a centred fractional spline that does not have this drawback. Let $\alpha=n+v$. We define a fractional $\alpha$-order B-spline as the function that has

$B_{0}^{\alpha}(\omega)=\left[\frac{\sin (\omega / 2)}{\omega / 2}\right]^{n+1} \cdot\left|\frac{\sin (\omega / 2)}{\omega / 2}\right|^{v}$

as FT. When $\alpha$ is an integer, $v=0$ and we obtain the normal $n$-order Bspline, while when $v \neq 0$, we obtain a fractional centred and symmetric B-spline that is the convolution of two even functions. To obtain $\beta_{0}^{\alpha}(\mathrm{t})$, we only have to convolve (4.4) with (4.14). Instead of performing this convolution, it is preferable to proceed recursively by successive convolutions with $\beta_{0}^{0}(\mathrm{t})$.

$\beta_{0}^{\mathrm{v}+\mathrm{n}}(\mathrm{t})=\beta_{0}^{\mathrm{v}+\mathrm{n}-1}(\mathrm{t}) * \mathrm{~b}_{0}^{0}(\mathrm{t})$

In performing such recursions we have to compute the following kind of primitives in $\tau$ :

$I_{\tau}|t-\tau|^{\theta}=-\frac{|t-\tau|^{\theta+1} \operatorname{sgn}(t-\tau)}{(\theta+1)}$

and

$I_{\tau}|t-\tau|^{\theta} \operatorname{sgn}(t-\tau)=-\frac{|t-\tau|^{\theta+1}}{(\theta+1)}$

With these primitives we can compute recursively the successive convolutions referred above. It is a somehow fastidious but not difficult computation. We are led to:

$\beta_{0}^{\mathrm{v}+\mathrm{n}}(\mathrm{t})=$

$\sum_{k=-\infty}^{+\infty} b_{k} \sum_{m=0}^{n+1}\left(\begin{array}{c}n+1 \\ m\end{array}\right)(-1)^{m}\left|t-m-k+\frac{n+1}{2}\right|^{v+n} \operatorname{sgn}^{n+1}\left(t-m-k+\frac{n+1}{2}\right)$

where we wrote as $\gamma$ the constant factor in (4.14) and

$b_{k}=\frac{\gamma \cdot(-1)^{k}}{(v)_{n+1} \Gamma(v / 2+k+1) \Gamma(v / 2-k+1)}$ 
In the following figures we present some splines for values of $n=0,1$, and 2 and $v=0.1,0.3,0.5,0.5$, and 0.9 .

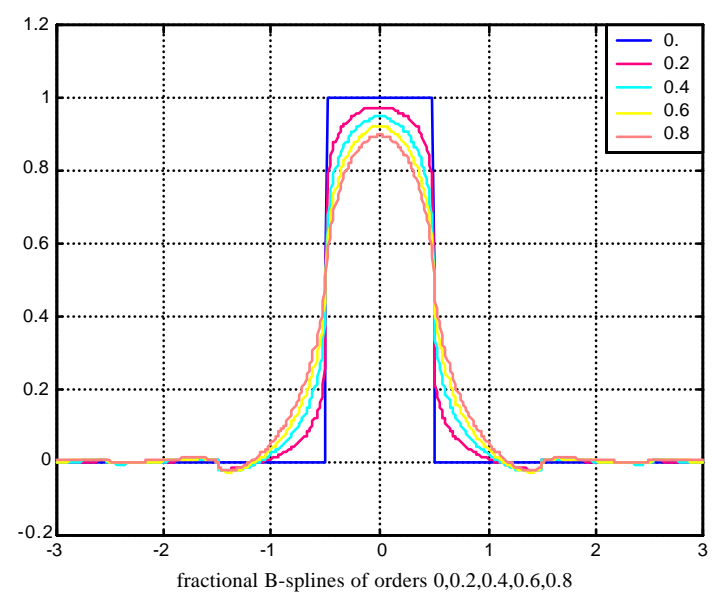

Figure 5 - Some fractional B-splines for $0 \leq \alpha<1$.

As it can be seen, the fractional B-splines interpolate the integer ones and become sooth as the order increases.

\section{Conclusions}

In this paper, we presented an approach into Fractional Calculus having in mind generalisations of wellknown concepts currently used in Signal Processing. We presented the differintegration based on Laplace Transform. With this definition we introduced the Transfer Function that we used to convert a continuous-time fractional linear system to a discrete-time form. We proposed two ways of performing this transform, one based on the backward difference transformation and the other based on the bilinear transformation. With this we could propose a new Grünwald-Letnikov integral. At last, we presented a fractional symmetric B-spline generalising a well-known concept.

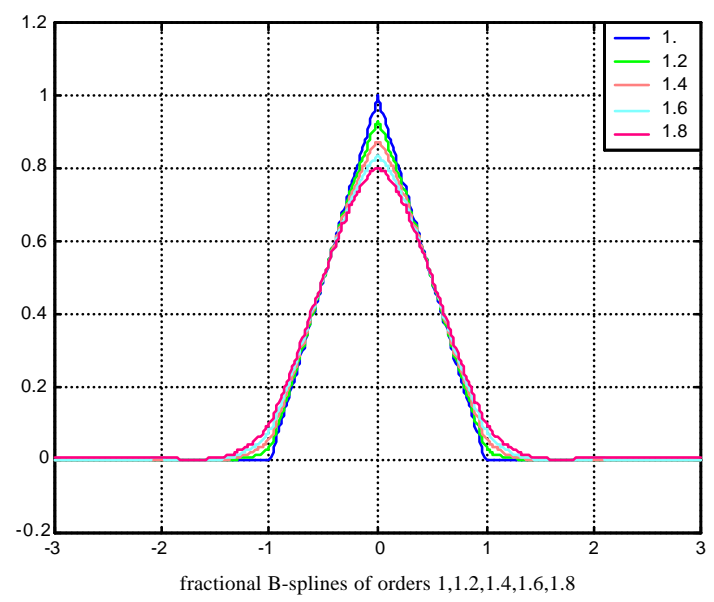

Figure 6 - Some fractional B-splines for $1 \leq \alpha<2$.

\section{REFERENCES}

[1] Ambardar, A., "Analog and Digital Signal

Processing”, PWS Publishing, 1999.

[2] Campos L. M. C., "On a Concept of Derivative of Complex Order with Applications to Special

Functions," IMA Journal of Applied Mathematics, 33, 109-133, 1984.

[3] Ferreira, J. C., "Introduction to the Theory of distributions", Pitman Monographs and Surveys in Pure and Applied Mathematics, July 1997.

[4] Henrici, P., "Applied and Computational Complex Analysis," Vol.II, Wiley, 1977

[5] Kalia, R. N. (Ed. ) "Recent Advances in Fractional Calculus," Global Publishing Company, 1993.

[6] Miller, K.S. and Ross B., "An Introduction to the Fractional Calculus and Fractional Differential Equations", Wiley, 1993.

[7] Machado, J.A.T. "Analysis and Design of Fractional-Order Digital Control Systems", SAMS, vol. 27, 107-122, 1997.

[8] Matignon, D. and d' Andréa-Novel, B., "Décompos. modale fractionaire de l'équation des ondes avec pertes viscothermiques", Tech. Rep. 95 C 001, École Nation. Sup. des Télécommunications, 1995.

[9] Nishimoto, K., "Fractional Calculus", Descartes Press Co., Koriyama, 1989.

[10] Ortigueira, M. D. "Introd. to Fractional Signal Processing. Part 1: CT Systems", IEE Proc. On Vision, Image and Signal Processing, 1, Feb. 2000.

[11] Ortigueira, M. D. "Introd. to Fractional Signal Processing. Part 2: DT Systems", IEE Proc. On Vision, Image and Signal Processing, 1, Feb. 2000.

[12] Podlubny, I., "Fractional Differential Equations", Academic Press, 1999.

[13] Samko, S.G., Kilbas, A.A., and Marichev, O.I., "Fractional Integrals and Derivatives - Theory and Applications," Gordon and Breach Science Publishers, 1987.

[14] Unser, M., " Splines: a perfect fit for Signal/Image Processing," IEEE Signal Processing Magazine, Vol.16, No.6, 22-38, Nov. 1999.

[15] Unser, M. and Blu, T., "Fractional Splines and Wavelets", SIAM Review, Vol. 42, 1, 43-67, 2000.

[16] Vinagre, B. M., Chen, Y. Q., and Petrás, I., "Two Methods for Direct Discretization of Fractionalorder Differentiator/Integrator," Submitted for publication in Automatica, January, 2001. 